\title{
WILLOQ: UN CASO DE TURISMO VIVENCIAL*
}

\author{
Alfredo Abarca \\ ALUMNO DEL PROGRAMA MAGÍsTER EN \\ DIRECCIÓN DE EMPRESAS DE ESAN (PERÚ) \\ MACZ0101@esan.edu.pe
}

\section{Resumen}

La comunidad de Willoq y Peruvian Odyssey han unido esfuerzos para acercar a los turistas a la vida cotidiana de una comunidad altoandina de las localidades de Ollantaytambo en el departamento del Cusco, lo que se conoce como turismo vivencial, pero con la idea clara de mantener y preservar las costumbres del hombre del Ande. Quizá la magia y el encanto que experimentan los visitantes de Willoq resida tanto en el vasto y diverso paisaje que se expande a su vista como en las diversas manifestaciones de una cultura viva tan diferente a la suya en cuanto a: organización, economía, religión e interacción sostenida a lo largo de los tiempos con su medio ambiente. Además, en Willoq se puede observar la cruda realidad de las dificultades que afrontan sus pobladores, así como la ilusión y la esperanza con la que ven un mundo cada vez más globalizado.

Palabras clave: turismo vivencial, responsabilidad social, desarrollo comunitario, comunidad nativa, Perú, Cusco, estudio de caso.

\begin{abstract}
The Willoq community and Peruvian Odyssey have joined efforts in order to bring the everyday life of the high Andean communities in the area of Ollantaytambo closer to tourists. This is known as "existential tourism», and has as a basic premise the preservation of the Andean Man's customs and traditions. Perhaps the magic and charm experienced by those who visit the Willoq community resides in the vast and diverse landscape in front of them, as well as in the various cultural manifestations that are so different to theirs in terms of: organization, economy, religion, and a sustainable interaction through time with the environment. But we can also see the crude reality and difficulties of the Willoq community, as well as the illusion and hope with which they view an ever more globalized world.
\end{abstract}

Key words: existential tourism, social responsibility, community development, native community, Peru, Cusco, case study.

* Una versión anterior de este caso fue premiada en el Primer Concurso de Casos sobre Responsabilidad Social organizado por la Universidad ESAN, con auspicio de la Asociación Atocongo, en el primer semestre de este año (2005). 
$\mathbf{A}_{\mathrm{d}}$ 45 minutos de recorrido en carro desde la localidad de Ollantaytambo $^{1}$, luego de atravesar paisajes de espectacular belleza, se llega a Willoq, comunidad campesina que es visitada por turistas extranjeros desde el año 1992, gracias a la visionaria y pionera iniciativa de Marco Bustamante Arenas, gerente general de la agencia Peruvian Odyssey. En efecto, Marco escuchó con atención la propuesta que le hacía Justino Melo, experimentado guía y cocinero de su agencia de viajes, de incluir a esta comunidad entre los atractivos que ofrecían a los turistas.

Justino Melo era también un diligente promotor y gestor para el desarrollo de Willoq, y consideró que una buena opción para este pueblo era convertirse en un nuevo destino turístico para la agencia Peruvian Odyssey, a cambio de lo cual podría recibir ayuda para hacer frente a sus graves carencias, especialmente en salud y educación.

\section{Peruvian Odyssey}

Peruvian Odyssey, agencia de viajes y turismo, fue fundada el 14 de marzo de 1984 en la ciudad de Lima por Daniel Taramona y Jodi Anderson Robinson. Tres años después, la agencia fue adquirida por el actual propietario y gerente, Marco Bustamante, quien buscando expandir las operaciones de la empresa abrió inmediatamente una sucursal en el Cusco. Con ello se convirtió en la primera agencia en el ámbito nacional en administrar y dirigir sus operaciones desde la propia Ciudad Imperial.

Marco Bustamante, peruano, casado con Alcione Vera y padre de Karina, Michel y Paola, nació en Santa Rosa, provincia de Melgar, departamento de Puno, pero desde pequeño vivió en el Cusco. Estudió la primaria y la secundaria en el colegio Inca Garcilaso de la Vega y luego siguió la carrera de Turismo en la Universidad San Antonio Abad del Cusco. Hábil y de espíritu emprendedor, fue docente destacado de su alma máter, hizo estudios de posgrado en turismo en España y después desempeñó cargos directivos en la agencia de turismo que finalmente compró a sus propietarios originales. Su conocimiento del negocio lo llevó a asumir el reto de manejar su propia empresa.

\section{Productos turísticos de Peruvian Odyssey}

\begin{tabular}{ll}
\hline Destinos turísticos & Servicios \\
Cusco y Machu Picchu & Programas largos: 21 días \\
Norte del Perú (e Islas Galápagos) & Programas medios: 8 días \\
Reserva Nacional de Tambopata & Programas cortos: 4 días \\
Ecuador & Extensión de programas o circuitos \\
Arequipa y valle del Colca & adicionales \\
Bolivia & Caminata y montañismo \\
\hline
\end{tabular}

[www.peruvianodyssey.com].

1. Antigua ciudadela inca, uno de los destinos turísticos de mayor preferencia. Mantiene in- tacta la estructura urbana y la distribución de las casas desde la época prehispánica. 
Nadie podría negar que lo ha hecho muy bien. Peruvian Odyssey obtuvo el premio a la Mejor Agencia de Viajes y Turismo durante tres años consecutivos: 2001, 2002 y 2003, en reconocimiento a sus programas sociales y a la calidad de sus servicios.

En una entrevista concedida a la revista Rumbos $^{2}$, le preguntaron acerca del nuevo destino que su empresa estaba ofreciendo y que cada vez se hacía más conocido. A lo que Marco respondió:

Sí, desde 1992 Peruvian Odyssey ha estado llevando visitantes a Willoq, una comunidad indígena del Ande, en el valle del río Patacancha. Todas las semanas nuestros turistas tienen la oportunidad de visitar esta comunidad y experimentar lo que ellos describen como «la mejor parte» de su viaje al Perú. Ahora muchos otros operadores han comenzado a seguir nuestro ejemplo y nosotros siempre advertimos a los involucrados a respetar ambos aspectos, la cultura y el medio ambiente de estos destinos.

\section{Justino Melo}

Referirse a Justino Melo Echami es referirse a la historia de Willoq y su desarrollo reciente como importante destino turístico. Justino es natural de Ollantaytambo y desde niño tuvo la suerte de viajar a las comunidades altas situadas a lo largo del cauce del río Patacancha, acompañando a sus padres, comerciantes y trabajadores del campo. En estos viajes conoció a mucha gente, especialmente de las comunidades de Willoq y Patacancha.

2. Rumbos. Lima, 2002, vol, 6, n. ${ }^{\circ}$ 32, pág .116 [http://www.rumbosdelperu.com].
Justino realizó sus estudios primarios y secundarios en el Colegio Mixto de Ollantaytambo. Desde escolar se sintió atraído por la cocina y empezó a trabajar en un restaurante turístico de la localidad. Cuando cursaba el tercer año de secundaria profundizó en la técnica y los conocimientos necesarios para la preparación de comida andina y criolla; de esta forma alternó los estudios con el trabajo. Al terminar la secundaria, decidió que definitivamente su futuro estaba entre ollas y peroles, por lo que se preocupó de asistir a cuanto curso de cocina le era posible -siempre sobre potajes andinos- tanto en el Cusco como en Lima.

Como parte de su formación, Justino hizo también cursos de primeros auxilios, entre los que él recuerda especialmente uno realizado en la localidad de Huaraz. Finalmente, participó en sendos cursos sobre orquídeas y plantas medicinales.

Paralelamente a su desarrollo profesional como cocinero, Justino fue dedicándose a una actividad que le recordaba los años de su infancia y que él considera uno de los grandes aciertos de su vida: las caminatas. Justino ha emprendido innumerables viajes de reconocimiento e identificación de nuevas rutas para ofrecerlas como destinos para el turismo de aventura en el Perú. Por esta labor y sus deseos de superación, es muy respetado y querido por los pobladores de las comunidades de Willoq y de todo el valle de Patacancha. Ni qué decir de sus compañeros de trabajo y su jefe, quienes le guardan un reconocimiento bien merecido.

En 1992, Justino se sumó al equipo de Peruvian Odyssey, con lo cual hizo realidad sus sueños de trabajar en una empresa importante y en las actividades que 


\section{Cusco, destino turístico}

A pesar de que el Perú cuenta con una diversidad de recursos turísticos -históricos, paisajísticos, ecológicos, entre otros- Cusco, el centro administrativo del famoso Imperio de los Incas, sigue siendo el destino turístico más importante del país. Ubicado en la zona sur de los Andes peruanos, en sierra y ceja de selva, ofrece al visitante un asombroso conjunto de restos arqueológicos que no cesan de descubrirse (el último de ellos, Choquequirao), así como el legado de su proceso de mestizaje con lo hispano, que en lo cultural y religioso se traduce en valiosas piezas de arte (pintura, escultura, entre otras), una notable arquitectura y un folclor que causan admiración. De su pasado prehispánico quedan zonas arqueológicas no menos admirables. Entre las más conocidas están las cercanas Sacsayhuaman, Tambomachay, Kenco y Puca-Pucara, también las del llamado Valle Sagrado, como Ollantaytambo, Písac y Chinchero. No obstante, el mayor atractivo es la ciudadela de Machu Picchu, también llamada la Ciudad Perdida de los Incas. Hace algunos años, la excursión a pie, desde el Cusco hasta Machu Picchu siguiendo el Camino Inca, se ha convertido en uno de los mayores atractivos de los amantes del turismo de aventura.

tanto le satisfacen y que mejor sabe hacer. En una nueva faceta de su carrera, asumió la responsabilidad de dirigir el área de turismo de aventura, lo que comprende organizar y coordinar los viajes a Machu Picchu por el Camino Inca, realizar viajes de inspección y reconocimiento de nuevas rutas y también, por supuesto, coordinar la visita de los turistas a la comunidad de Willoq.

Al principio, las visitas a la comunidad no fueron fáciles, dada la desconfianza y resistencia de los pobladores a recibir gente extraña de quienes no sabían qué podían esperar. Cierta vez, cuenta Justino, los guías de otra agencia de turismo condujeron a un grupo de turistas a Willoq, pero a mitad del camino les salieron al encuentro el presidente de la comunidad y varios comuneros para impedirles el acceso. La excursión fue cancelada, con la consiguiente frustración de los turistas, y la agencia debió asumir el costo de su improvisación. Los guías regresaron con estas palabras retumbando en sus oídos: «Ustedes nunca nos han ayudado en nada...».
Para conducir a un grupo de turistas a Willoq, Justino tiene previamente que conversar con las juntas directivas de la comunidad, tanto de varones como de mujeres, y solicitar la debida autorización para que la delegación de Peruvian Odyssey pueda ingresar sin inconvenientes a la comunidad e incluso visitar los hogares y participar en las labores que realizan los pobladores. La intervención de Justino ha sido vital en el desarrollo del destino turístico Willoq, y aunque este paso inicial fue decisivo, el mayor desafío ha sido integrarse a la comunidad para entender sus problemas y ayudarla a superarlos.

\section{La comunidad de Willoq}

Willoq es una comunidad campesina perteneciente al distrito de Ollantaytambo, provincia de Urubamba, departamento del Cusco. Está ubicada a $17 \mathrm{~km}$ subiendo a lo largo de la cuenca del río Patacancha, a una altura de 3400 m.s.n.m. Desde que se empieza el ascenso, el turista se siente transportado a otra dimensión, a un mundo diferente preservado del tiempo, cual 


\section{Estructura organizacional de la comunidad de Willoq}

La dirigencia de la comunidad de Willoq está organizada en una junta directiva formada por: presidente, vicepresidente, secretario, tesorero y fiscal. La junta directiva es la encargada de tomar las decisiones en aquello que concierna a la comunidad y de solucionar los problemas que puedan afectarla. Sólo los varones pueden formar la junta directiva. El presidente actual para el periodo 2005-2006 es Crisóstomo Sinchi Puma. La comunidad de Willoq también tiene alcaldes y regidores. El alcalde llamado de derecha representa a la parte baja de la comunidad, la principal. Antonio Echame ejerce este cargo actualmente. EI alcalde llamado de izquierda representa a la parte alta de la comunidad (alrededores). Mario Medina ejerce este cargo actualmente. Los alcaldes se encargan de coordinar las festividades, visitar otras comunidades y organizar las asambleas generales. Cada uno de ellos tiene seis regidores, quienes pueden ser niños desde 4 años de edad hasta jóvenes de 18 años de edad. Las mujeres de la comunidad también tienen su junta directiva. Se organizan para tratar temas referidos a sus tejidos (combinación de colores, diseños, entre otros aspectos). También organizan "pasantías», esto es, intercambio de ideas y experiencias con mujeres de otras comunidades.

si estuviera en la época de la floreciente cultura incaica. El sol ilumina los cerros y los sembríos de papa, habas verdes, maíz, oca, quinua, tarhui, todos irrigados por las cristalinas aguas que vienen desde el pantanal de Yauricunca, ubicado en la parte alta de la comunidad. Asombra la riqueza de flora y fauna: 60 especies de aves y más de 700 especies de plantas.

Lo que más impresiona, sin embargo, es la gente, la cultura viva, expresada en cada rostro. La tradición impregna todas las tareas y actividades; desde el niño inquieto hasta el adulto maduro, los pobladores han sabido practicar y conservar las sabias técnicas del cultivo de la tierra. Las diestras mujeres dominan el tejido en telar y confeccionan sus vestimentas. En la estructura del gobierno de la comunidad se incluye a los niños, porque estos no mienten; el brazo derecho del presidente tiene que ser un niño.

Willoq pertenece a un grupo étnico muy particular de la sierra peruana. Según los escritos de los cronistas, mantie- ne una descendencia directa de los últimos incas, lo cual puede ser corroborado por los apellidos actuales de la gente del lugar, como Yupanqui, Sinchi, Pukha, Huamán, Cusipáucar, Tupha, entre otros.

Pero visitar Willoq es también conocer sus problemas. Marcia Fernández, administradora de la oficina de Peruvian Odyssey en la ciudad del Cusco, comentó lo siguiente luego de su primer viaje a la comunidad:

Los niños, tan tiernos, dejan ver sus ojos expresivos, pero el resto de sus caritas está lleno de sarna ${ }^{3}$; y sus cabellos negros parecen castaños de tantos piojos que tienen alojados en sus cabecitas. En general, son de contextura delgada y tienen el rostro demacrado.

Esta impresión resumía el terrible contraste entre, por un lado, la belleza pai-

3. Sarna: Enfermedad contagiosa producida por el arador o ácaro. Consiste en multitud de vesículas y pústulas diseminadas por el cuerpo que causan vivo escozor. 


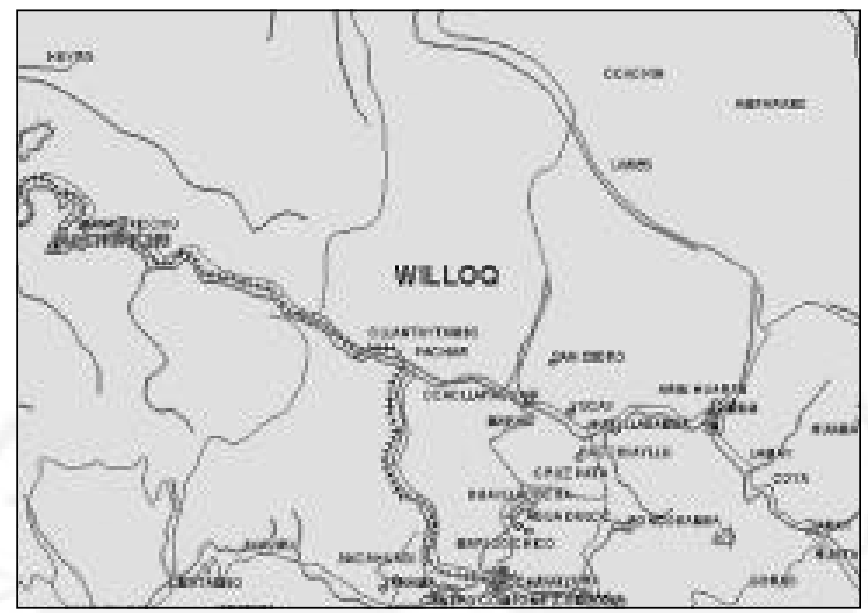

sajista, la originalidad y colorido de las vestimentas, la forma de vida ligada a la naturaleza y, por otro, la precaria calidad de vida de los pobladores. Según las estadísticas mostradas en el anexo 1, la situación de higiene y salud era uno de los principales problemas de la comunidad. La mayoría de hogares únicamente contaba con una habitación, la que era utilizada como cocina, comedor y dormitorio para toda la familia, en promedio de seis personas.

\section{Los wayruros ${ }^{4}$ de Willoq, 25 años como porteadores del Camino Inca}

Aproximadamente a inicios de los años ochenta, los wayruros de Willoq comen-

4. Se les llama wayruros (semilla pequeña de color rojo y negro) por el color rojo de la vestimenta. El término denota a todos los miembros de la comunidad de Willoq, incluso a los de otras comunidades por esta típica y colorida vestimenta.

5. Que tiene oficio de portear, llevar o conducir de una parte a otra una cosa por un pago convenido. En el Camino Inca, los porteadores llevan los equipos y víveres de los turistas desde zaron a trabajar como porteadores ${ }^{5}$ del Camino Inca. El pago por el servicio que prestan es convenido previamente y depende de la agencia de viajes y de la temporada. En muchas ocasiones, cuando la demanda de porteadores disminuye, estos tienen que bajar sus precios obligados por la falta de empleo. Marcia Fernández explica:

En la actualidad algunas agencias pagan 80 soles por cuatro días de trabajo, sin incluir los pasajes de ida y vuelta en tren; estos corren por cuenta del porteador. Además, la comida que les proporcionan en el viaje es muy pobre, no es suficiente para que puedan recuperar energías y estar óptimos al día siguiente. Si los porteadores llegan a culminar su trabajo es porque ellos llevan consigo sus hojas de coca y van piqchando $^{6}$ en el camino. Terminado el viaje tienen que esperar semanas y a veces meses para que estas agencias les paguen por su trabajo. Realmente es un abuso...».

el kilómetro 88 hasta el Santuario de Machu Picchu.

6. Masticar la hoja de coca y mantenerla en la boca. 
No obstante, las agencias agrupadas a la Asociación de Agencias de Turismo del Cusco (AATC) han fijado la tarifa por los cuatro días en 125 soles. Peruvian Odyssey forma parte de esta asociación y, además de la suma correspondiente, entrega a los porteadores una propina adicional como incentivo por su apoyo a la empresa. Asimismo, les compra los pasajes de tren y los boletos de ingreso, les proporciona alimentación completa durante todo el viaje (incluida chicha de jora) y les paga por sus servicios el mismo día que terminan el Camino Inca (Justino mismo lo hace). En todo sentido, se les brinda mejor trato que en otras agencias.

Un aspecto que no puede dejar de mencionarse es que al tener a Willoq como uno de sus productos turísticos más atractivos, Peruvian Odyssey debe cumplir con los acuerdos tomados con la población en cuanto a los pagos. Si no se les pagara a los porteadores al término de la caminata, la siguiente visita a Willoq estaría condicionada a la deuda pendiente. Por ello, podemos observar que los pobladores deben estar involucrados en el desarrollo de nuevos destinos turísticos.

Los porteadores proceden de distintas localidades de las partes altas de todo el Valle Sagrado de los Incas y están inscritos como personal de apoyo en los padrones de las distintas agencias. Peruvian Odyssey cuenta con 30 porteadores inscritos en su padrón de personal y todos ellos pertenecen a los wayruros de Willoq.

Justino se reúne con todos los porteadores en el mes de marzo de cada año para coordinar el trabajo que se realizará durante los meses en que los turistas demandan la excursión por el Camino Inca, esto es, en la temporada seca, de abril a setiem- bre (ver anexo 2). En tiempo de lluvias no se oferta el Camino Inca por los peligros que entrañan las condiciones climáticas desfavorables.

Todos los porteadores son empleados durante los meses en que se realiza la excursión por el Camino Inca, ninguno se queda sin prestar servicios porque Justino se encarga de elaborar un cronograma que permite rotar a todos los integrantes de la nómina, en estrecha coordinación con los representantes de los wayruros de Willoq.

Marcia nos dice que:

De los treinta porteadores que tenemos, dos son jefes de porteadores; cuatro, «carperos», llamados así porque cargan y arman las carpas; y dos, asistentes de cocina. El cocinero principal es Justino. Para el Camino Inca proporcionamos dos porteadores por pasajero. Cada porteador debe cargar veinte kilos de peso como máximo.

\section{Peruvian Odyssey y la comunidad de Willoq: una relación «ventajosa»}

Puede decirse que la relación establecida entre empresa y comunidad ha ido muy ventajosa en este caso. Peruvian Odyssey brinda ayuda a la comunidad de Willoq en tres aspectos fundamentales: salud, educación y cultura.

\section{Salud}

En salud, se desarrollan campañas de prevención y atención (curación) de enfermedades comunes y temporales: infecto-contagiosas propias de los niños, como la sarna y la pediculosis (piojos), e infecciones broncopulmonares, por ejemplo. 
Las visitas se realizan dos veces por año con la participación de médicos y enfermeras, quienes llevan todo el equipamiento necesario. También se dan charlas de orientación a los niños sobre temas de higiene y salud y la empresa entrega una dotación de medicinas básicas para la implementación del botiquín escolar.

La decidida intervención de Peruvian Odyssey para tratar de resolver la problemática de Willoq ha generado gran confianza en los pobladores. Ante cualquier eventualidad recurren a la agencia para solicitar ayuda, especialmente cuando se trata de hospitalizaciones u operaciones. Entre los muchos casos presentados, cabe hacer mención al de la bebe Lidia Echame, de un mes de nacida, quien aquejada de una broncopulmonía que casi le cuesta la vida, estuvo hospitalizada una semana. La empresa intervino también para hacer posible la operación de labio leporino del niño Mariano Laucada, de tres años y medio de edad. Finalmente, se recuerda la ayuda prestada a la señora María Quispe, una tejedora diestra y muy trabajadora que, al acudir a una faena convocada por la comunidad, sufrió un accidente de trabajo y se fracturó el brazo derecho. La agencia se hizo cargo de la situación una vez más y corrió con los gastos de atención de la señora en una clínica especializada.

Peruvian Odyssey participa también en la lucha contra el alcoholismo en coordinación con las autoridades comunales. Se han emprendido campañas de prevención y sensibilización -con la ayuda de fotos y videos- acerca de los graves problemas que ocasiona esta enfermedad dentro de la familia y la comunidad, además de constituir un freno para el desarrollo de Willoq. El alcoholismo, del que tanto varones como mujeres son víctimas, ha tenido trágicas consecuencias para la comunidad, pues dos de sus miembros murieron en grescas producidas tras borracheras. Descontando los efectos dañinos del alcohol para la salud y las relaciones sociales, también conviene referirse al impacto negativo sobre la identidad cultural de Willoq, ya que los pobladores han estado olvidando la costumbre de consumir la conocida chicha, elaborada a partir del maíz.

\section{Educación}

En cuanto a la educación, uno de los principales problemas de la comunidad era la falta de personal docente, por lo que la empresa ha contratado y paga a un profesor desde hace más de cinco años para que se haga cargo del aula multigrado del centro educativo San Juan Bautista de la Salle N. ${ }^{\circ}$ 50617. Para complementar esta labor, Peruvian Odyssey entrega a los niños materiales didácticos correspondientes a diferentes grados, útiles escolares y artículos deportivos.

Preocupada por el desarrollo integral de los niños, Peruvian Odyssey ha contribuido a la formación de la banda escolar. Obsequió al colegio diferentes instrumentos musicales típicos de la zona, como pitos, tinyas, pinkullos y tambores, de manera que los niños han participado durante dos años consecutivos en el desfile por el Día del Cusco. Así, han tomado conciencia de que son los embajadores de su cultura en los diferentes escenarios y actividades donde se han presentado.

En diversas oportunidades, la empresa brinda a los niños del centro educativo la oportunidad de viajar a la ciudad del Cusco para que estén en contacto con otras realidades y conozcan los atractivos turís- 
ticos de la capital del Incanato. También se entrevistan con autoridades municipales, visitan el zoológico de la universidad, parques infantiles, el aeropuerto, entre otros lugares.

En fechas especiales, como el Día del Estudiante y la Navidad, Peruvian Odyssey organiza reuniones de camaradería, juegos deportivos y chocolatadas, durante las cuales los niños reciben juguetes y pasan gratos momentos.

\section{Cultura}

Peruvian Odyssey es consciente de la pérdida de los valores culturales que viene sufriendo la comunidad de Willoq, por lo que desarrolla diversas actividades con el fin de revalorar lo tradicional.

En Willoq se está promoviendo la confección de sombreros y monteras, prendas que hacen tan especial al poblador de la zona. Estas prendas han estado siendo reemplazadas por gorras y sombreros modernos, completamente extraños a la vestimenta de los wayruros. En la comunidad no había personas que supieran confeccionar sombreros, por lo que la agencia, en coordinación con las autoridades comunales, organizó talleres de confección de estos artículos, tanto para autoconsumo como para su venta en las ferias dominicales. Los talleres se dictaron para las mujeres (monteras) y también para los varones (sombreros) y estuvieron a cargo de especialistas de la zona de Chumbivilcas y Chinchero -lugares de donde proceden los antiguos sombreros-, quienes también proporcionaron los materiales e instrumentos necesarios.

Con el mismo objetivo de salvaguardar los elementos culturales de la comu- nidad a la vez que proporcionar a los pobladores un medio de obtención de ingresos, la empresa propició la creación y reconocimiento de la Asociación Artesanal Qori Awaq Warmikuna, formada por las mujeres de Willoq con la finalidad de mejorar la producción de artesanías y lograr una mayor participación en la venta de sus productos a los turistas.

Para que las poblaciones que se encuentran en las cercanías de la cuenca del río Patacancha (Willoq, Tastayoc, Patacancha, Cachin, Qelcanca, Yanamayo, Pallata, entre otras) tengan facilidades de accesibilidad y estén debidamente comunicadas, Peruvian Odyssey, en convenio con otras instituciones y la Municipalidad de Ollantaytambo, ha hecho posible el mejoramiento y la habilitación de la carretera que une a estas poblaciones rurales andinas.

Se viene implementando un proyecto para la revalorización y cultivo de la maca, producto preincaico extinguido en la zona en la época colonial. Hoy se sabe que esta planta constituye una fuente importante de proteínas y que bien podría enriquecer la alimentación de los habitantes de esta comunidad. Este es un convenio tripartito entre Willoq, el Instituto Nacional de Investigación Agraria (INIA) y Peruvian Odyssey.

De igual manera, las tres instituciones mencionadas están promoviendo la crianza de cuyes, animales que actualmente complementan la alimentación de los habitantes pero en pequeñas cantidades.

Justino observó que la televisión en la comunidad debería estar prohibida a los niños para que estos no copien formas de vida y de vestir de otras realidades. «La 
televisión está prohibida a los niños en Willoq...», dice Marcia. Sin embargo, también señala que a pesar de que constantemente se les dice a los pobladores que deben mantener su forma original de vestimenta, muchas veces los adultos de la comunidad se han presentado en las oficinas de la agencia vestidos con casacas, gorros y pantalones, a la usanza occidental. Esta situación es clara consecuencia del contacto directo entre los porteadores y los turistas, quienes en su afán de ayudarlos acostumbran regalarles algunas prendas de vestir que los porteadores lucen gustosos. La agencia no tiene el control sobre el $100 \%$ de los porteadores de la comunidad, quienes prestan sus servicios a distintas agencias.

Se han dictado charlas de capacitación a los porteadores para que puedan mejorar su desempeño. Algunos de los temas desarrollados han sido técnicas de campamento, primeros auxilios y manejo de artesanías.

\section{Problemática}

Peruvian Odyssey sigue ofreciendo la comunidad de Willoq como destino turístico con mucho éxito. Otras agencias hacen lo mismo, tratando de realizar labores de apoyo y participar activamente en el desarrollo sostenido de la comunidad de Willoq y de todos los destinos turísticos, porque ¿quiénes más que los involucrados directamente en la industria del turismo para cuidar la fuente de sus ingresos y la de las futuras generaciones?

En el aspecto humano, se ha hecho muy bien en apoyar a una comunidad de nuestra serranía, sumida en problemas de calidad de vida, agobiada por la pobreza y olvidada en esta era de la globalización. Hoy existe esperanza y oportunidades para los campesinos y sus hijos. Quizá sin el apoyo directo del sector privado la miseria se habría incrementado hasta provocar un descontento generalizado y protestas sociales.

Los mayores logros de Peruvian Odyssey han sido en el aspecto de la salud. Se ha reducido significativamente el porcentaje de niños que padecen de sarna o piojera; estas enfermedades prácticamente han sido controladas. Sin embargo, las campañas de prevención continúan para evitar cualquier rebrote.

El alcoholismo está siendo enfrentado con mucha responsabilidad por los mismos comuneros, quienes tratan el tema permanentemente en las asambleas generales. Esta batalla la están ganando los comuneros de Willoq.

En el campo educativo, se sigue proporcionando útiles, libros y cuadernos, y eventualmente se contrata profesores para el desarrollo de actividades específicas, como música, artesanía, elaboración de sombreros, entre otras.

El aspecto cultural ofrece puntos críticos y discutibles, pues Willoq ha cambiado su forma de vida. Ahora la principal actividad es el turismo, tanto para los porteadores como para las mujeres tejedoras, lo que poco a poco ha causado que los lugareños descuiden sus cotidianas labores agrícolas. Su dependencia del turismo es grande y también lo es del apoyo permanente de las agencias de viajes, situación que hace vulnerable a la comunidad frente a un eventual cambio desfavorable para la actividad turística. 
La globalización, la interrelación con los turistas y su vínculo con el mercado están occidentalizando a Willoq; será una tarea difícil mantener la tradicional forma de vida de los lugareños. La televisión y la radio han ingresado a sus viviendas y los efectos negativos de estos medios de comunicación se dejan sentir sobre todo en los niños, que buscan imitar todo lo que ven.

En la actualidad, el trato abusivo de algunas agencias hacia los porteadores se ha minimizado gracias a la organización y protestas continuas de ellos mismos, quienes en varias oportunidades han venido en marchas de protesta a la ciudad del Cusco. El mayor logro ha sido conseguir estandarizar la retribución mínima por sus servicios para todas las agencias: 125 soles por cuatros días, además de la alimentación y los pasajes. Quizá esto no habría sido posible si agencias como Peruvian Odyssey -por su respeto a los porteadores- no hubieran establecido tarifas por encima del promedio del sector.
Para esto también ha sido decisiva la cada vez mayor intervención de las entidades estatales para regular el sector turístico y evitar la proliferación de agencias irresponsables que explotan a los prestadores de servicios y, muchas veces, estafan a los turistas.

Después de más de diez años de labor en Willoq, Peruvian Odyssey, su gerente, Justino y el personal administrativo se preguntan si en verdad lograron sus objetivos, si la responsabilidad social de la empresa frente a la comunidad y a la sociedad en general estuvo bien orientada. ¿Valió la pena el esfuerzo desplegado? ¿Cómo sería Willoq si Peruvian Odyssey dejaría de operar como agencia de viajes? ¿Deben continuar con su política actual o es momento de replantearlo todo?

¿Usted, como experto en desarrollo, qué opina? ¿Ha actuado adecuadamente Peruvian Odyssey o hay algunas fisuras en su enfoque de responsabilidad social? 


\section{Anexo 1 \\ Características socio-demográficas y de vivienda de la comunidad campesina de Willoq}

Departamento: Cusco

Provincia: Urubamba

Distrito: Ollantaytambo

\section{Demográficas}

1.Población

Mujeres

2.Grupos de edad

Menores de 1

De 1 a 4

De 5 a 14

De 15 a 64

De 65 a más

3.Migración

Nativos

Migrantes

Extranjeros

4.Minusválidos

Con ceguera

Con retardo o alteraciones mentales

Con polio

Invalidez de extremidades inferiores

Invalidez de extremidades superiores

Otros

\section{Educativas}

1.Población analfabeta según sexo

Hombres

Mujeres

2.Nivel educativo

Sin nivel

Inicial preescolar

Primaria

Secundaria

Superior

\section{Laborales}

1. PEA de 6 a 14 años

2. PEA de 15 y más años de edad 
Desocupados

3.Ocupación principal

Agricultores y trabajadores calificados agrícolas

Obreros de manufactura, minas, construcción y otros

Comerciantes al por menor

Vendedores ambulantes

Trabajadores no calificados de servicios (excluidos vendedores ambulantes)

Otros

4.Categoría ocupacional

Asalariado

Independiente

Patrono

Trabajador familiar no remunerado

Trabajador del hogar

5.Actividad económica

Extractiva

Transformación

Servicios

\section{Características sociales}

1.Estado civil

Conviviente

Casado (a)

Soltero (a)

Otros

2.Jefatura de hogar

Hombre

Mujer

3.De la mujer

Promedio hijos por mujer (40 a 49 años)

Mujeres de 15 a 49 con más de 4 hijos

Madres solteras (12 a 49 años)

- De 12 a 19

- De 20 a 29

- De 30 a 49

Madres adolescentes (12 a 19 años)

\section{Del hogar y vivienda}

Total de viviendas

Total de hogares

1.Tamaño promedio del hogar

2.Tipo de vivienda (ocupadas y desocupadas)

Casa independiente

Vivienda improvisada

Otros

3.Tenencia de la vivienda (ocupadas)

Alquilada 
Ocupada de hecho

Otros

4.Material de construcción de la vivienda

Paredes

- De ladrillo o bloque de cemento

- De quincha

- De piedra con barro

- De madera

- De estera

- Otros

0

0

Techo

- De concreto armado

- De plancha de calamina o similares

- De estera con torta de barro

- De paja, etc.

- Otro material

5.Servicios de la vivienda

Abastecimiento de agua

- De red pública dentro y fuera de la vivienda

- Pilón de uso público

- Camión-cisterna o similar

- Otros

Servicio higiénico conectado a:

- Red pública dentro y fuera de la vivienda

- A pozo ciego o negro

- Otros

- No tiene

Alumbrado eléctrico

- Sí tiene

- No tiene

6.Viviendas con sólo una habitación

\section{Características del hogar}

Sin habitación exclusiva para dormir

Con uso de servicio higiénico compartido

Con espacio para actividad económica

8.Equipamiento del hogar

Sin artefacto electrodoméstico $\quad 50$

$\begin{array}{ll}\text { Con sólo radio } & 65\end{array}$

Con sólo radio, TV B/N o color $\quad 72$

Con máquina de coser $\quad 0$

Con refrigeradora $\quad 0$

Con triciclo para trabajo $\quad 0$

Con 4 y más artefactos electrodomésticos $\quad 0$

Fuente: INEI. IX Censo de Población y IV de Vivienda 1993. 


\section{Anexo 2}

Procedencia de los huéspedes no residentes en el Perú que arribaron a los establecimientos de hospedaje: Cusco, abril del 2005

\begin{tabular}{|c|c|c|}
\hline País o región & $\begin{array}{c}\text { Distribución } \\
\text { porcentual (\%) }\end{array}$ & $\begin{array}{c}\text { Promedio de } \\
\text { permanencia (días) }\end{array}$ \\
\hline Argentina & 2,39 & 2,34 \\
\hline Alemania & 5,28 & 2,25 \\
\hline Bolivia & 0,32 & 2,99 \\
\hline Brasil & 2,60 & 2,29 \\
\hline Canadá & 3,97 & 2,38 \\
\hline Centroamérica & 0,47 & 2,11 \\
\hline Colombia & 0,86 & 2,14 \\
\hline Chile & 0,89 & 2,21 \\
\hline Ecuador & 0,46 & 2,30 \\
\hline Estados Unidos & 24,60 & 2,36 \\
\hline España & 3,35 & 2,20 \\
\hline Francia & 11,14 & 2,12 \\
\hline Italia & 2,79 & 2,37 \\
\hline Japón & 5,56 & 1,91 \\
\hline Corea del Norte & 0,49 & 1,34 \\
\hline México & 1,75 & 2,35 \\
\hline Paraguay & 0,33 & 2,22 \\
\hline Inglaterra o Reino Unido & 10,49 & 2,27 \\
\hline Uruguay & 0,22 & 2,34 \\
\hline Venezuela & 0,36 & 2,31 \\
\hline África & 0,29 & 2,22 \\
\hline Oceanía & 4,92 & 2,27 \\
\hline Otros países de América & 0,38 & 2,34 \\
\hline Otros países de Asia & 0,46 & 2,65 \\
\hline Otros países de Europa & 12,01 & 2,31 \\
\hline India & 0,20 & 2,23 \\
\hline Israel & 2,45 & 2,63 \\
\hline Corea del Sur & 0,53 & 3,18 \\
\hline República Popular China & 0,37 & 2,69 \\
\hline Singapur & 0,08 & 2,41 \\
\hline Total & 100,00 & 2,28 \\
\hline Total de arribos & 33285 & \\
\hline
\end{tabular}

Fuente: Ministerio de Comercio Exterior y Turismo (Mincetur). Estadísticas de arribos. Abril 2005 [www.mincetur.gob.pe]. 